\title{
INTERVENÇÃO FISIOTERAPÊUTICA ASSOCIADA A ACUPUNTURA NA PARALISIA FACIAL PERIFÉRICA: um relato de caso
}

Carlos Dornels Freire de SOUZA ${ }^{1}$

Lucas Carvalho de MACEDO

Victor Lucas Batista NASCIMENTO²

Marcus Armando Fernandes da SILVA ${ }^{1}$

Denilson José de OLIVEIRA $^{1}$

Bruna Ângela ANTONELLI ${ }^{1}$

${ }^{1}$ Fisioterapeutas. Professores do Colegiado de Fisioterapia da Faculdade São Francisco de Juazeiro- FASJ, Juazeiro, Bahia, Brasil.

${ }^{2}$ Acadêmicos do curso de Fisioterapia da Faculdade São Francisco de Juazeiro- FASJ, Juazeiro, Bahia, Brasil.

Recebido em: 20/09/2016 - Aprovado em: 04/12/2016 - Disponibilizado em: 18/12/2016

\section{RESUMO:}

Introdução. A Paralisia Facial Periférica é uma síndrome clínica de etiologia incerta, resultante da lesão do nervo facial. Objetivo. Relatar a intervenção fisioterapêutica associada a acupuntura em indivíduo com paralisia facial periférica, atendido no Laboratório Multifuncional da Faculdade São Francisco de Juazeiro, no período entre março e junho de 2016. Método: Trata-se de um relato de caso. Inicialmente, realizou-se a anamnese e o exame físico do paciente, usando o sistema de classificação de House-Brackmann. Após a avaliação, foi traçado um plano terapêutico composto por 50 sessões de fisioterapia, realizadas diariamente, exceto em finais de semanas e feriados. O programa foi composto por eletroterapia com corrente Aussie, massoterapia, cinesioterapia, Facilitação Neuromuscular Proprioceptiva e acupuntura. Resultados. Após o tratamento, o paciente foi reavaliado. Quanto a classificação de House-Brakmann, a função motora foi classificada como normal, com alinhamento do ângulo da boca, capacidade de fechamento palpebral alternado e sem lagoftalmo, de enrugamento da testa, de assovio e de sugar usando canudo. Conclusões. A abordagem fisioterapêutica proposta associada a acupuntura mostrou-se eficaz na recuperação neuromotora do paciente com paralisia facial periférica.

Palavras-chave: Fisioterapia, paralisia facial, acupuntura, terapia por estimulação elétrica.

\section{PHYSIOTHERAPEUTIC INTERVENTION ASSOCIATED WITH ACUPUNCTURE IN PERIPHERAL FACIAL PARALYSIS: a case report}

\begin{abstract}
:
Introduction: Facial Paralysis Peripheral is a clinical syndrome of unclear etiology, resulting from facial nerve injury. Objective: Report physiotherapy intervention associated with acupuncture in individual with peripheral facial paralysis, attended at Laboratório Multifuncional da Faculdade São Francisco de Juazeiro, between March and June 2016. Metodology. This is a case study. Initially, were held anamnesis and physical examination of the patient, using HouseBrackmann scale. After evaluation, a therapeutic plan was traced composed of 50 physiotherapy sessions, daily performed, excluding weekends and holidays. The program was composed by electrotherapy with Aussie current, massotherapy, kinesiotherapy, proprioceptive neuromuscular facilitation and acupuncture. Results: After treatment, the patient was reevaluated. As to House-Brackmann scale, motor function was graded as normal, with alignment angle of the mouth, alternating eyelid closure capacity and without lagophthalmos, of forehead wrinkle, of whistle and suck using straw. Conclusion: The physiotherapeutic approach proposed associated with acupuncture proved effective in neuromotor recovery of patient with peripheral facial paralysis.
\end{abstract}

Keywords: Physiotherapy, facial paralysis, acupuncture, Electric stimulation therapy 


\section{Introdução}

A paralisia facial periférica é uma síndrome clínica de etiologia incerta, resultante da lesão do VII par craniano - nervo facial, podendo, a lesão, situar-se em qualquer ponto do seu trajeto, desde o núcleo petrotuberancial à junção neuromuscular, ou até mesmo pela lesão de todo o nervo. ${ }^{1,2} \mathrm{O}$ ponto de corte para caracterização das paralisias faciais é o núcleo do nervo facial, sendo que as lesões acima são caracterizadas como centrais, acometendo a porção inferior da hemiface contralateral, e as lesões infranucleares são caracterizadas como periféricas, acometendo toda a hemiface ipsilateral. ${ }^{3-4}$

O nervo facial é um nervo misto, cujas fibras motoras correspondem a $70 \%$, porção denominada de nervo facial propriamente dito, e as fibras sensitivas corresponde a $30 \%$, porção denominada de nervo intermédio ou de Wrisberg. ${ }^{1,3}$ Após emergir do sulco bulbopontino, os dois componentes penetram pelo meato acústico, local onde as fibras se juntam, formando um tronco nervoso único, que após uma série de curvaturas emergirá do crânio pelo forame estiloide. Ao atravessar a glândula parótida, emitirá uma série de ramos que inervarão os músculos da mímica facial. ${ }^{1,5}$

$\mathrm{Na}$ maioria das vezes (cerca de $80 \%$ dos casos), ocorre recuperação espontânea do quadro, podendo demorar de algumas semanas a alguns anos. O tempo e o grau de recuperação dependerão de um conjunto de fatores, tais como a idade do paciente, do tipo de lesão, da etiologia da paralisia, do grau de nutrição do nervo, do grau de comprometimento muscular e do tratamento estabelecido.,

Quinze a vinte por cento dos casos desenvolverão sequelas motoras, sendo as mais comuns as sincinesias, contraturas musculares, ausências de linhas de expressão, rima nasolabial pronunciada, elevação da comissura labial e asa do nariz e assimetria do sorriso. ${ }^{7}$ Além dos danos funcionais, destacam-se os prejuízos psicológicos e sociais, como o isolamento. ${ }^{8}$

O tratamento da $\mathrm{PB}$ deve envolver uma abordagem multiprofissional, incluindo médicos, fisioterapeutas, fonoaudiólogos e psicólogos. Estudos recentes têm destacado a importância da combinação de diferentes estratégias terapêuticas a fim de facilitar o processo de recuperação e reduzir o risco de ocorrência de sequelas. ${ }^{9,} 10$

Como base no exposto, este trabalho teve como objetivo demonstrar, através de um estudo de caso, a importância da reabilitação fisioterapêutica associada à acupuntura na paralisia facial periférica, como estratégia para a recuperação funcional precoce e redução do risco de desenvolvimento de sequelas. 
Método

\section{Descrição do Caso}

Paciente RFS, sexo Masculino, 28 anos de idade, residente e natural do município Juazeiro-Bahia. Na história da doença atual, relatou que em 06 de março de 2016 notou dormência na cavidade oral e língua. No dia seguinte, já apresentou perda motora na hemiface esquerda. Consultado o neurologista, foi diagnosticada a Paralisia Facial Periférica idiopática. Na ocasião, foi prescrito Aciclovir 200mg e Prednisona 20mg.

Em 15 de março procurou o serviço de fisioterapia da Faculdade São Francisco de Juazeiro, com queixa de dificuldade para sorrir, alimentar-se e realizar autocuidados. O voluntário informou não ter feito exames complementares para a identificação da etiologia da paralisia.

Nessa primeira consulta, além da anamnese, foi realizado o exame físico e funcional da face e classificação da paralisia segundo o sistema de House-Brackmann. Vale ressaltar que o voluntário assinou o Termo de Consentimento Livre e Esclarecido (TCLE), autorizando a realização do tratamento fisioterapêutico, sendo-lhe garantido o direito de desistir da intervenção a qualquer tempo.
No exame, verificou-se paralisia em toda hemiface esquerda (ausência de expressão facial), associada a hipotonia muscular, lagoftalmo, sinal de Bell, desvio do ângulo oral para a direita, disartria e perda gustativa. $\mathrm{O}$ reflexo corneano estava preservado.

Para a avaliação da função motora adotou-se uma ficha composta por um conjunto de questões que revelavam a função dos músculos da expressão facial (tabela 1). Nela observamos os músculos, os comandos verbais adotados e o estado dos músculos avaliados (orbicular dos olhos, frontal, corrugador do supercílio, orbicular da boca, elevador do lábio superior e do ângulo da boca, zigomático, risório, abaixador do ângulo da boca e do lábio inferior e bucinador).

Foi adotada a classificação da paralisia facial segundo o sistema de HouseBrackmann, da Academia Americana de Otorrinolaringologia, que varia entre grau I (função facial normal em todas as áreas) até o grau VI (paralisia total). A paralisia do paciente foi classificada como grau V (disfunção importante/severa). ${ }^{11}$ No geral, o grau V caracteriza-se pela presença de uma movimentação discretamente perceptível; ao repouso, apresenta assimetria; e ao movimento, não se observa nenhum movimento na testa, o fechamento dos olhos é incompleto e a boca tem movimento discreto. 
Tabela 1. Avaliação da função motora do paciente.

\begin{tabular}{c|c|c}
\hline Músculo & Comando verbal & Resultado \\
\hline Orbicular dos olhos & Feche os olhos & Paralisia à esquerda \\
\hline Frontal & Levante a sobrancelha & Paralisia à esquerda \\
\hline Corrugador do supercílio & $\begin{array}{c}\text { Traga a sobrancelha para } \\
\text { baixo juntas }\end{array}$ & Paralisia à esquerda \\
\hline Orbicular da boca & Feche e enrugue a boca & Paralisia à esquerda \\
\hline $\begin{array}{c}\text { Elevador do Lábio } \\
\text { superior e elevador do } \\
\text { ângulo da boca }\end{array}$ & $\begin{array}{c}\text { Levante o lábio superior } \\
\text { Zigomático }\end{array}$ & Paralisia à esquerda \\
\hline Risório & Levante o canto da boca & Paralisia à esquerda \\
\hline $\begin{array}{c}\text { Abaixador do ângulo da } \\
\text { boca e abaixador do lábio } \\
\text { inferior }\end{array}$ & $\begin{array}{c}\text { Abaixe o ângulo da boca } \text { os } \\
\text { Bucinador }\end{array}$ & Paralisia à esquerda \\
\hline & $\begin{array}{c}\text { Sopre enchendo as } \\
\text { bochechas }\end{array}$ & Paralisia à esquerda à esquerda \\
\hline
\end{tabular}

Fonte: Autores

Após a avaliação neuromotora, foi definida a abordagem fisioterapêutica adotada. O tratamento foi realizado entre 23 de março e 09 de junho de 2016, no laboratório multifuncional do departamento de Fisioterapia da Faculdade São Francisco de Juazeiro-FASJ. Foi adotado um protocolo intensivo, composto por 50 sessões (sendo 05 por semana, exceto feriados), com duração aproximada de 50 minutos, cada. Ao final desse período, foi realizada a reavaliação neuromotora.

O programa terapêutico proposto conjugou eletroterapia, recursos terapêuticos manuais (RTM), cinesioterapia, Facilitação Neuromuscular Proprioceptiva (FNP) e acupuntura. A proposta iniciou com a aplicação da corrente Aussie, modo sincrônico (rise:2s, on:4s, decay:2s e off:5s), carrier de 1khz, duração de Burts de 2ms, frequência de Burts de $40 \mathrm{hz}$ e duração de 20 minutos. Foram utilizados quatro eletrodos (tamanho aproximado de $2 \times 2 \mathrm{~cm}$ ), sendo dois pontos na hemiface superior e dois na hemiface inferior.

Em seguida, foi empregado um conjunto de exercícios, associados a estímulos manuais de FNP, com o objetivo de ativação e reeducação dos músculos da mímica facial: fechar os olhos com força, elevar as sobrancelhas, franzir a sobrancelha e o nariz, inflar as bochechas, esboçar sorriso aberto e fazer "bico”. Na medida em que os músculos apresentavam resposta, novos exercícios eram incluídos, tais como assoviar, sugar líquidos usando canudos e encher balões de gás. Ao final das sessões, foram realizadas massagens na hemiface sadia, utilizando bola tipo cravo, 
com a finalidade de promover relaxamento dessa musculatura.

\section{Resultados}

Por fim, foram feitas orientações para o domicílio: fechar a pálpebra com as mãos e massageando a musculatura envolvida, uso de tapa olho para dormir e a realização de exercícios nos dias em que não havia sessão.

A acupuntura foi incluída a partir de 20 de abril (um mês após o início do tratamento), sendo uma sessão semanal, totalizando 7 sessões, com duração de aproximada de 20 minutos, cada. A acupuntura teve como objetivo retirar vento nos canais presentes no rosto. Foram utilizados os pontos seguintes pontos: Vesícula biliar - 14 (Yangbai), homolateral à afecção; 20 (Fengchi) bilateral; estômago - 2 (Sibai), 3 (Juliao), 4 (Dicang), 6 (Jiache), todos homolateralmente à afecção; vaso governador - 16 (Fengu); intestito grosso 4 (Hegu), nomolateral à afecção; efígado 3 (Taichong), Contra-lateral à afecção.

Em 09 de junho foi realizada a reavaliação funcional. Quanto à classificação de House-Brackmann após a intervenção, a função motora na hemiface esquerda foi classificada como normal. Foi observada melhora significativa na função dos músculos responsáveis pelas expressões faciais (figura 1), demonstrada pelo alinhamento do ângulo da boca em repouso e no sorriso, capacidade de fechamento palpebral alternado e sem lagoftalmo, capacidade de enrugamento da testa, de assovio e de sugar usando canudo. Evidenciou-se, também, a melhora da capacidade gustativa e da articulação das palavras.

Figura 1. Aspectos da musculatura facial antes e após o tratamento fisioterapêutico.

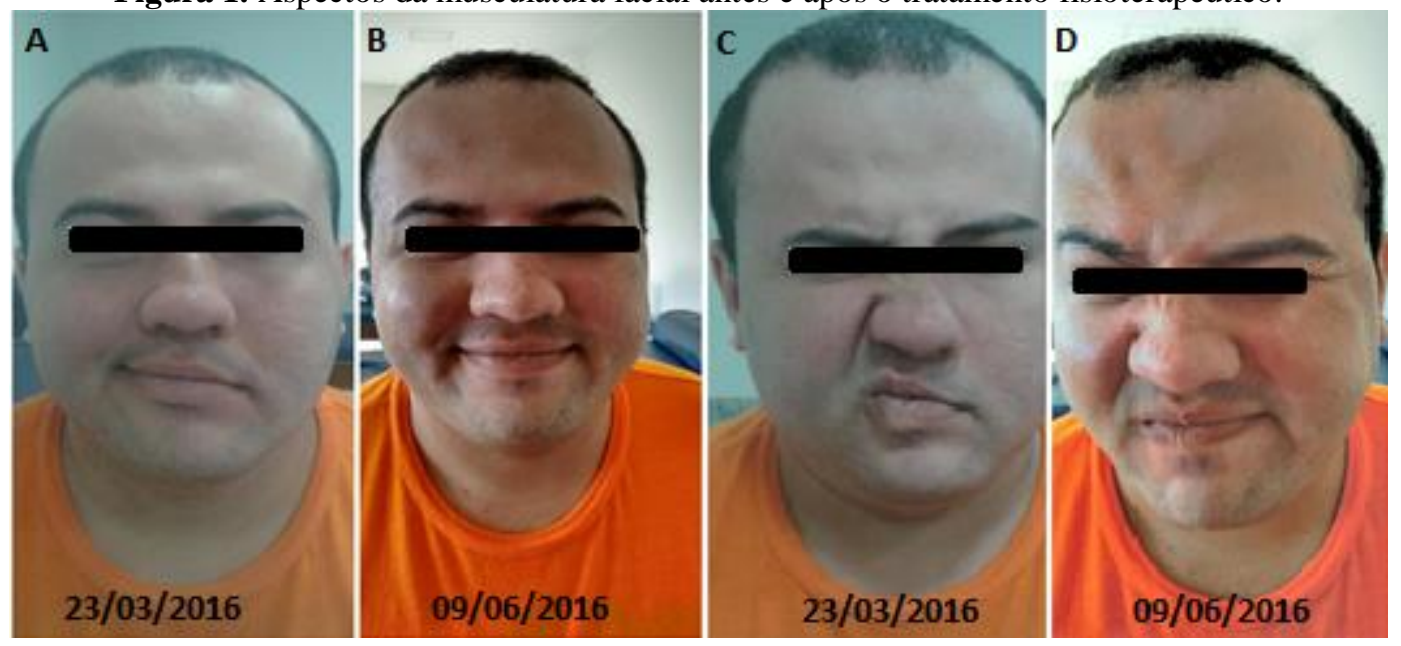

Fonte: Autores

Legenda: Na figura A, pré-intervenção, observamos o desvio do ângulo oral para a direita. Na figura B, pós intervenção, observação alinhamento e simetria do ângulo oral e capacidade de sorrir sem exposição os dentes (risório). $\mathrm{Na}$ figura $\mathrm{C}$, observamos a paralisia da musculatura da hemiface esquerda quando solicitada a realização da expressão de "Mal cheiro". Já na figura D, podemos observar a ativação da toda a musculatura da hemiface esquerda. 


\section{Discussão}

A fisioterapia tem sido uma grande aliada no tratamento da paralisia facial periférica, sendo fundamental para recuperação precoce das funções neuromotoras da face e redução do risco de ocorrência de sequelas. O rol de métodos, técnicas e recursos fisioterapêuticos tem possibilitado a elaboração de programas terapêuticos mais dinâmicos e efetivos.

No estudo de revisão de Souza et $a l^{12}$, foram selecionados 10 artigos, dentre eles estudos de casos, estudos longitudinais e experimentais, referentes as condutas terapêuticas que contribuem para o tratamento da PFP. Dentre os recursos e instrumentos utilizados destacam-se as terapias manuais, a cinesioterapia, a eletrotermofototerapia e os treinamentos funcionais.

Os artigos citados no estudo ainda ressaltaram que a PFP ultrapassa o limite da doença física, levando ao comprometimento psicológico e social. Por essa razão, é destacada a importância de uma equipe multiprofissional no acompanhamento dos pacientes com PFP, tais como médicos, fisioterapeutas, psicólogos e fonoaudiólogos.

Ainda segundo a autora, dentre os recursos fisioterapêuticos incluem estimulação elétrica, massagens, exercícios faciais e reeducação neuromuscular isolados, ou combinações desses e, ainda, variação de parâmetros para um mesmo recurso. No estudo de caso apresentado, o programa terapêutico foi elaborado incluindo diferentes recursos.

O uso da Facilitação Neuromuscular Proprioceptiva (FNP) tem sido utilizada por possibilitas o retorno da precoce da funcionalidade da musculatura facial, ainda mais quando associada a cinesioterapia. Em um estudo de caso realizado por Lima et $a l^{13}$, a FNP foi utilizada como recurso terapêutico em uma paciente com diagnóstico clínico de paralisia facial periférica há oito anos e diagnóstico cinético - funcional de paresia facial bilateral. Foram realizadas 20 sessões e após, foi possível observar o fechamento ocular com ausência do fenômeno de Bell, abolição da perda de líquido através da comissura labial, além de modulação do tônus e incremento da força muscular. Percebeu-se ainda que a FNP contribuiu de forma eficaz para o retorno da funcionalidade da mastigação e da deglutição dos alimentos, bem como da articulação da fala.

Segundo Adler et $a l^{14}$, técnicas de estiramento rápido e de resistência possibilitam o incremento da atividade muscular e da força. Os contatos manuais têm função tanto de guiar os movimentos como de facilitá-los. É importante ter atenção aos contatos, pois quando errados acabam facilitando o aparecimento de sincinesias e padrões motores inadequados.

Os autores ressaltam, ainda, que para se obter melhor resultado de força e otimização dos movimentos faciais, as sessões devem ser realizadas com maior frequência semanal. Por essa razão, o protocolo realizado neste estudo 
foi composto por sessões diárias, excetuando os finais de semana e feriados. A fisioterapia realizada de modo intensiva acelerou a recuperação funcional do paciente.

A literatura tem demonstrado que a massoterapia deve ter lugar de destaque no programa terapêutico, seja ela a clássica, a massagem das zonas reflexas, a do tecido conjuntivo e a dos pontos clássicos da acupuntura. Os principais benefícios evidenciados são o relaxamento muscular, a redução do quadro álgico, do edema e da estase linfática. ${ }^{15}$

Segundo Zineli ${ }^{16}$, a estimulação elétrica neuromuscular constitui-se num importante recurso no tratamento da paralisia facial, uma vez que favorece o fortalecimento muscular. Com o uso da eletroterapia, um conjunto de mudanças são induzidas nos músculos estimulados, tais como o aumento do metabolismo muscular, do da oferta de nutrientes e da remoção de subprodutos do metabolismo, favorecendo a recuperação funcional do músculo.

Embora a importância da eletroestimulação já seja demonstrada pelas pesquisas científicas, não há estudos sobre a utilização da corrente Aussie especificamente com o objetivo de reeducação motora e facilitação do reaprendizado motor em pacientes com PFP. No entanto, por apresentar duração de pulso curta, quando comparada com as correntes Russa e Interferencial Vetorial, torna a estimulação mais eficiente.
No caso aqui apresentado, a corrente mostrou bons resultados, todavia, carece de estudos mais específicos sobre os parâmetros a serem adotados.

A acupuntura foi incluída no tratamento a fim de potencializar os efeitos do processo de reabilitação. No estudo de Barros et $a l^{17}$, com o objetivo de acompanhar a evolução de uma paciente com paralisia facial periférica submetida ao tratamento com acupuntura, observou-se melhora significativa na escala de House-Brackmann, nos testes de função muscular da face e no potencial de ação muscular, avaliado por meio de eletroneuromiografia. Foram realizadas 20 sessões de acupuntura, sendo a avaliação feita antes do tratamento e após a $10^{\mathrm{a}}$ e a $20^{\mathrm{a}}$ sessão.

Uma revisão sistemática com metaanálise realizada por $\mathrm{Li}$ et $a l^{18}$, utilizando 14 ensaios clínicos com um total de 1.541 participantes, indicou que o uso da acupuntura na paralisia facial resulta em melhores respostas quando comparada a outras terapias.

\section{Conclusão}

Com base neste estudo, pode-se concluir que a fisioterapia se reveste de um papel fundamental na recuperação precoce das funções neuromotoras em indivíduos com paralisia facial, além de reduzir o risco do desenvolvimento de sequelas. A vasta gama de métodos e técnicas fisioterapêuticas possibilita 
a elaboração de programas terapêuticos polissêmicos e dinâmicos, capazes de potencializar o processo de reabilitação, sobretudo quando associados à acupuntura.

A combinação de recursos adotada neste estudo demonstrou ser eficaz na recuperação funcional completa deste paciente. Todavia, mais estudos devem ser conduzidos para analisar não só os efeitos de cada recurso terapêutico, como também da capacidade deles em evitar a ocorrência de sequelas.

\section{Referências}

1. MACHADO, A.B.M; HAERTEL, L.M. Neuroanatomia funcional. 3.ed. São Paulo: Atheneu, 2006.

2. KATO, Y; KAMO, H; KOBAYASHI, A; ABE, S; OKADA-OGAWA, A; NOMA, N. et al. Quantitative evaluation of oral function in acute and recovery phase of idiopathic facial palsy; a preliminary controlled study. Clin Otolaryngol. 2013;38(3):231-6.

3. VALENÇA, M.M; VALENÇA, L.P.A; LIMA, M.C.M. Paralisia Facial Periférica Idiopática de Bell: A propósito de 180 pacientes. Arq. Neuropsiquiatr. 2001; 59(3-B), 733-739.

4. RESENDE, L.A.L; WEBER, S. Peripheral facial palsy in the past. Arq Neuropsiquiatr. 2008; 66(3- B), 765-769.

5. MITRE, E.I; LAZARINI, P.R; DOLCI, J.E. Objective method for facial motricity grading in healthy individuals and in patients with unilateral peripheral facial palsy. Am J Otolaryngol. 2008;29(1):51-7.
6. VASCONCELOS, B.E.C; DIAS, E; DANTAS, W.R.M; BARROS, E.S, MONTEIRO, G.Q.M. Paralisia facial periférica traumática. Rev Cir Traumat

Buco-Maxilo-Facial. 2001;1:13-20.

7. WENCESLAU, L.G.C; SASSI, F.C; MAGNANI, D.M; ANDRADE, C.R.F. Paralisia facial periférica: atividade muscular em diferentes momentos da doença. CoDAS [online]. 2016;28(1):3-9.

8. TESSITORE, A; MAGNA, L.A; PASCHOAL, J.R. Medida angular para aferição do tônus muscular na paralisia facial. Pró-Fono R. Atual. Cient. [online]. 2010;22(2):119-124.

9. TEIXEIRA, L.J; SOARES, B.G; VIEIRA, V.P; PRADO, G.F. Physical therapy for Bell s palsy (idiopathic facial paralysis). Cochrane database of syst. Rev. 2008; 16(3), 6283 .

10. SALLES, A.G; TOLEDO, P.N; FERREIRA, M.C. Botulinum toxin injection in long-standing facial paralysis patients: improvement of facial symmetry observed up to 6 months. Aesthetic Plast Surg. 2009;33(4):582-90

11. House, J.W; Brackmann, D.E. Facial nerve grading system. Otolaryngol Head Neck Surg. 1985;93(2):146-7.

12. SOUZA, I.F. et al. Métodos Fisioterapêuticos utilizados no Tratamento da Paralisia Facial Periférica: Uma Revisão.

Revista Brasileira de Ciências da Saúde. 2015; 19(4): 315-320.

13. LIMA, F.S; FAGUNDES, D.S; LIMA, R.R.O. Facilitação neuromuscular proprioceptiva na reabilitação da paralisia facial periférica: um estudo de caso. Revista Científica da Faculdade de Educação e Meio Ambiente. 2016; 7(1): 27-40.

14. ADLER, S; BECKERS, D; BUCK, M. Facilitação neuromuscular 
proprioceptiva: um guia ilustrado. São Paulo: Manole, 1999.

15. BIENFAIT, M. Fáscias e pompages: estudo e tratamento do esqueleto fibroso. São Paulo: Summus, 1999. 67p.

16. ZINEZI, F.G. Comparação de dois protocolos de tratamento utilizando corrente de baixa frequência associado ou não a contração isométrica visando aumento da força muscular de preensão palmar em indivíduos saudáveis. Cascavel, 73 p., 2005. Trabalho de Conclusão de Curso - Curso de Fisioterapia da Unioeste, n.1, 2005.

17. BARROS, H.C; BARROS, A.L.S;

NASCIMENTO, M.P.R. Uso da Acupuntura no Tratamento da Paralisia Facial Periférica Estudo de Caso. Rev Neurocienc 2012;20(2):246-253.

18. LI, P; QIU, T; QIN, C. Efficacy of Acupuncture for Bell's Palsy: A Systematic
Review and Meta-Analysis of Randomized Controlled Trials. PLOS ONE. 2015;14;10(5):e01218. 